\title{
Origins Space Telescope cryo-thermal system
}

\author{
Michael DiPirro, ${ }^{a} *$ Louis Fantano, ${ }^{\text {a }}$ Thomas D'Asto, ${ }^{\text {b }}$ \\ Benjamin Gavares, ${ }^{c}$ and The Origins Study Team \\ ${ }^{a}$ NASA/Goddard Space Flight Center, Greenbelt, Maryland, United States \\ ${ }^{\mathrm{b}}$ ATA Aerospace, Greenbelt, Maryland, United States \\ ${ }^{\mathrm{c}}$ Northrop Grumman, Beltsville, Maryland, United States
}

\begin{abstract}
The Origins Space Telescope's orders-of-magnitude improvement over the scientific capabilities of prior infrared (IR) missions is based on its cold telescope $(4.5 \mathrm{~K})$ combined with low-noise far-IR detectors and ultra-stable mid-IR detectors. A number of trades were made in favor of a design with staged cooling, avoiding warm objects in the cold zones and minimizing the number of deployments and their complexity. The result is an architecture that is easily to analyze thermally and straightforward to test in existing facilities. (C) The Authors. Published by SPIE under a Creative Commons Attribution 4.0 Unported License. Distribution or reproduction of this work in whole or in part requires full attribution of the original publication, including its DOI. [DOI: 10 .1117/1.JATIS.7.1.011009]
\end{abstract}

Keywords: cryogenics; space telescope; cryocoolers; thermal analysis.

Paper 20097SS received Jul. 8, 2020; accepted for publication Dec. 1, 2020; published online Feb. 25, 2021.

\section{Introduction}

The Origins Space Telescope (Origins) study is one of four potential astrophysics flagship missions commissioned by NASA Headquarters for inclusion in the 2020 Astrophysics Decadal Survey. ${ }^{1}$ Origins goals covered imaging and spectroscopy over the wavelength range from 2.8 to $588 \mu$. The following describes the baseline point design that was used to determine feasibility, technology needs, and cost. A $4.5 \mathrm{~K}, 5.9-\mathrm{m}$ telescope is sky background-limited (i.e., background that is only limited due to celestial sources, and not sources within the observatory) over 3 to $250 \mu$ in the mid-to-far infrared wavelength range and only about a factor of 2.7 above the background at $600 \mu$. The instruments are state-of-the-art spectrometers and imagers in these wavelengths with advanced detectors that take advantage of the low background from the telescope. The thermal system passively and actively cools the telescope and instruments to $4.5 \mathrm{~K}$ and below.

The Origins design is driven by the cryogenic payload, testability, serviceability (both on the ground and on-orbit), and the minimization of deployments. The resulting architecture is more akin to the Spitzer Space Telescope ${ }^{2}$ (Spitzer) than the James Webb Space Telescope (JWST) ${ }^{3}$ (Fig. 1). Like Spitzer, Origins uses two deployable sunshield layers rather than a five-layer JWST design. Eliminating three deployable sunshield layers is possible because the telescope is surrounded by a barrel that is highly reflective toward the sunshield while radiatively cooling in the direction of deep space (Fig. 2). The sunshield also wraps around the bottom of the cryogenic payload, providing protection against the warm spacecraft, Sun, Earth, and Moon over a field of regard (FOR) 25\% larger than JWST's.

The resulting barrel temperature is $35 \mathrm{~K}$, Spitzer's was $34 \mathrm{~K}$. The barrel protects the colder surfaces within from higher temperature thermal radiation and lowers the heat load to be extracted by mechanical cryocoolers. The Spitzer scheme offers several advantages, including, the design is compact, requires few deployments, provides stray light protection, eases trade analyses by making the thermal design separable into subsystems inside and outside the barrel, and thermal performance is easier to verify on the ground. The two-layer sunshield is extremely easy to deploy compared with JWST and can be readily deployed, tested, and stowed on the ground. The sunshield shape was determined by a combination of the need to block Sun-, Earth-, and Moon-shine from the aperture and a desire to maintain as small a cross-section to solar

*Address all correspondence to Michael DiPirro, michael.j.dipirro@nasa.gov 


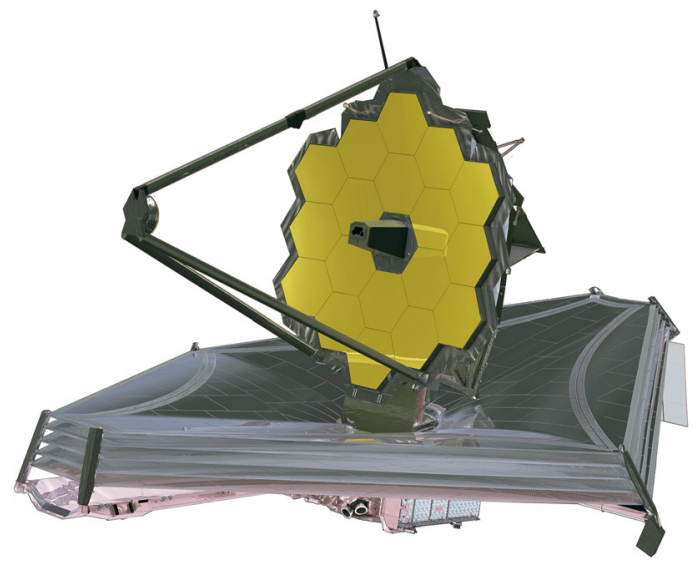

(a)

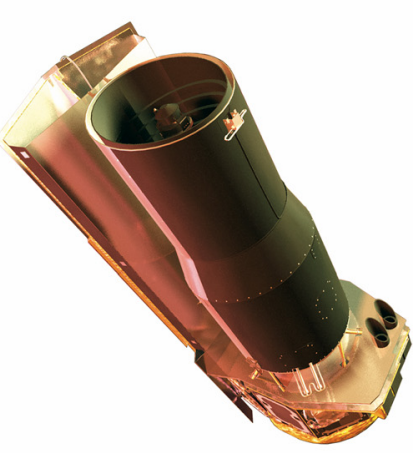

(b)

Fig. 1 The Origins architecture is more like (b) Spitzer than (a) JWST.

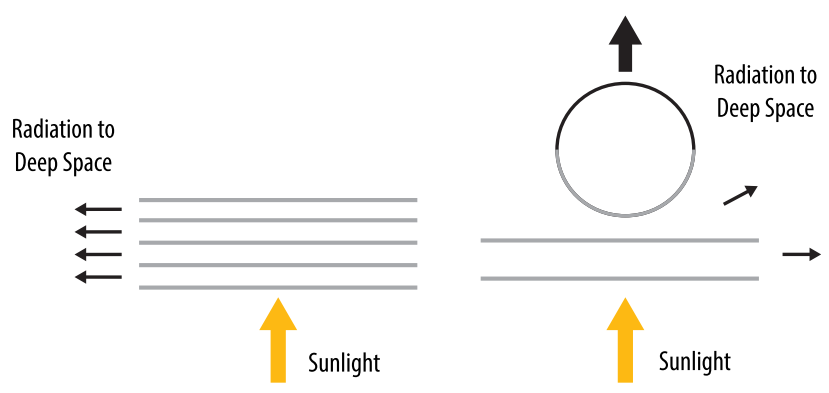

Fig. 2 A two-layer sunshield plus radiator can achieve the same radiative temperature as a fivelayer sunshield.

pressure as possible. The $35^{\circ}$ cut angle in the baffle, barrel, and sunshields keeps the Sun and Earth from shining into the baffle for the complete FOR for the entire orbit around Sun-Earth Lagrange Point 2 (SEL2). The large $~ 1.2-\mathrm{m}$ gap between the shields and the barrel allows efficient radiation to deep space, as shown in Fig. 3.

Four similar cryocoolers provide cooling to the observatory at three different temperatures: $4.5 \mathrm{~K}$ for the telescope, baffle, and instruments, $20 \mathrm{~K}$ as a radiative and conductive heat intercept between $35 \mathrm{~K}$ and $4.5 \mathrm{~K}$, and $70 \mathrm{~K}$ as a conductive heat intercept along the barrel bipods. While cooling is planned as a shared resource (cryocoolers all operate together, none are dedicated to a particular system), Origins has baselined four cryocoolers rather than one larger cryocooler

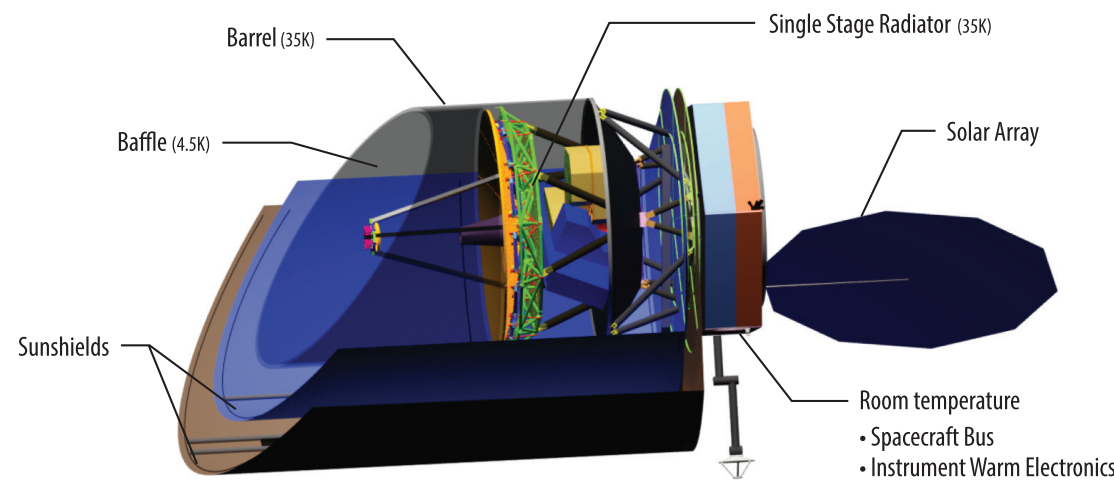

Fig. 3 Origins temperature zones are designed to maximize cryo-cooling efficiency while ensuring a $4.5 \mathrm{~K}$ environment for the telescope and instruments. The instruments are housed in the $4.5 \mathrm{~K}$ zone beneath the primary mirror. The $35 \mathrm{~K}$ radiator is more clearly shown in Fig. 5 . 
because the cryocooler size is closer to those already developed, ${ }^{4}$ if less cryocooling is required, it is easy to remove a cryocooler, and individual cooling heads may be positioned in several places on the telescope and instrument mounting surfaces to provide local heat sinks and minimize thermal straps.

An ejectable cover is included in the design for three purposes: to protect the mirrors during shipment and handling, to stiffen the barrel and baffle during launch, and to protect the sensitive telescope and detectors from exposure to the Sun during the early phase of the mission. After the first spacecraft thruster firing, the cover is ejected by a mechanism similar to that used on Spitzer.

\section{Details of the Configuration}

The overall Origins height is $12.7 \mathrm{~m}$ and overall diameter is $6.3 \mathrm{~m}$ in the launch configuration (Fig. 4). Origins launch configuration weight is 9.6 metric tons. This allows Origins to fit into the Space Launch System (SLS) Block II and Big Falcon Rocket launch vehicles. Once on orbit, Origins deploys its communication antenna, solar array, telescope cover, and sunshields. After deployments, the overall size of Origins is 12.7-m long and $\sim 9 \mathrm{~m}$ in diameter (Fig. 4). The colors in the figures simply show different components for clarity.

To be compatible with the SLS launch vehicle, the Origins observatory was stiffened until the normal modes were predicted to meet the requirements in the SLS User Guidelines. From the analysis of the Origins baseline design, the primary lateral and axial modes are predicted to be frequencies of 8.09 and $16.7 \mathrm{~Hz}$, respectively. These results meet the SLS User Guidelines, allowing simpler structural analysis going forward. To reach these frequency values, several design improvements were made: (1) the barrel and baffle were stiffened, (2) they are supported at the top at launch via the aperture cover, (3) extra struts were added from the spacecraft to the barrel, (4) the bottom of the barrel was made in the shape of a cone, and (5) the support of the propellant tanks was stiffened. These improvements added some mass and had some thermal impact, but result in a robust structural design without requiring a detailed coupled loads analysis. A system that thermally deployed the extra struts was briefly considered to lower the thermal impact, but a simpler, fixed structure that still met the thermal margins was selected instead.

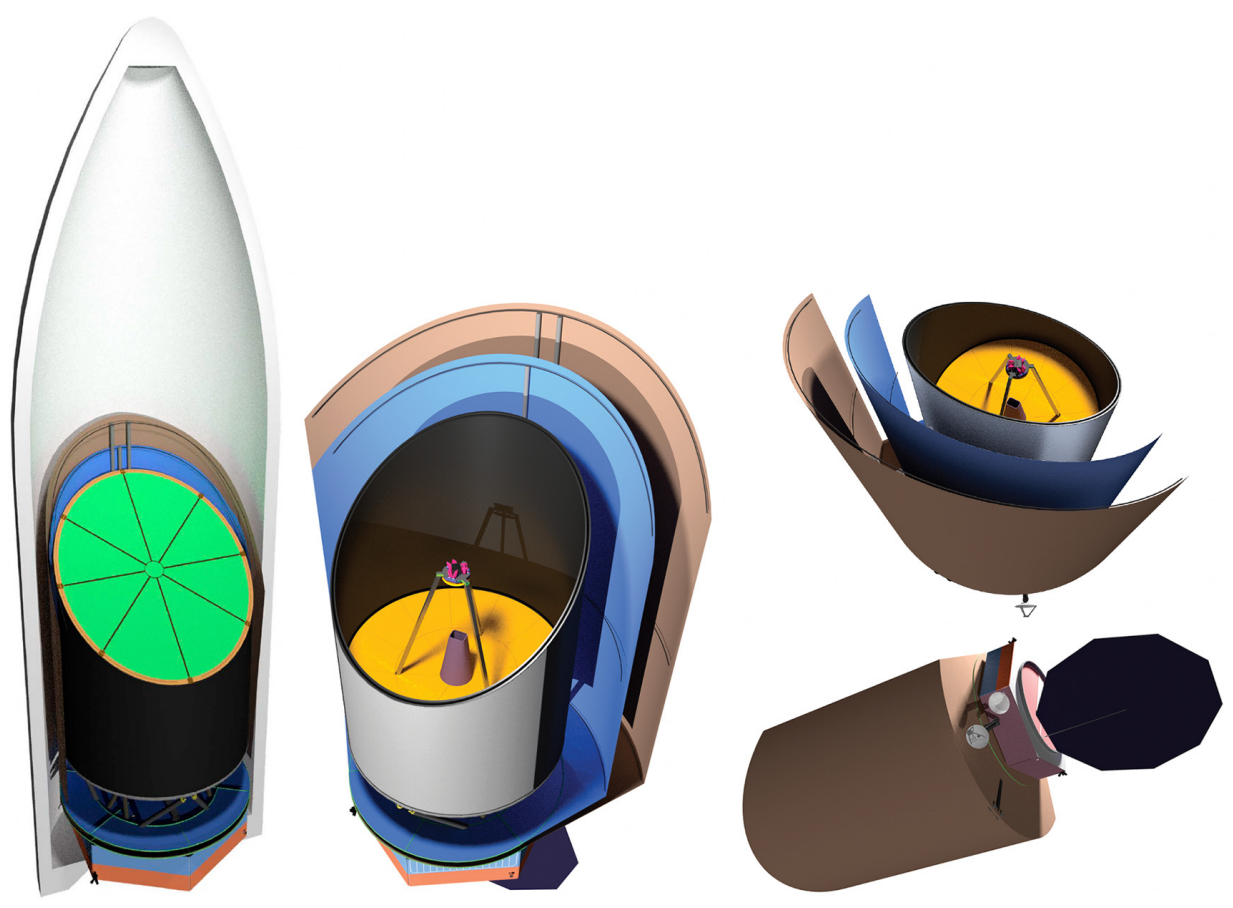

Fig. 4 The left side depicts Origins in the stowed configuration in the SLS fairing with sunshields wrapped around the barrel and ejectable cover, and the right side shows three different views of the fully deployed observatory. 


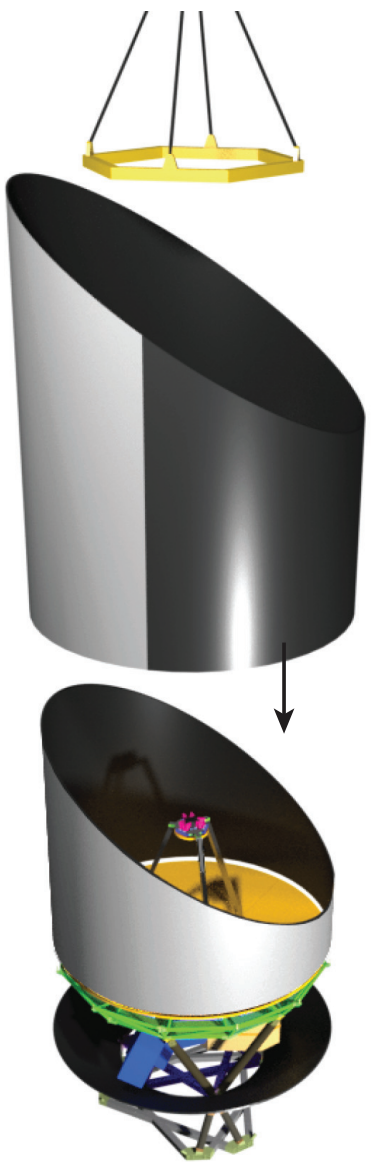

(a)

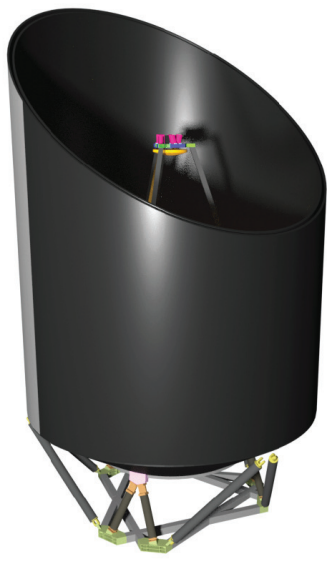

(b)

Fig. 5 (a) The Origins barrel is shown being lowered over the telescope and baffle assembly. (b) The assembly comprises the CPM, which can be structurally and thermally tested as a unit.

\subsection{Telescope and Instruments}

The telescope and instrument mounting structure reside at $4.5 \mathrm{~K}$, conductively cooled by the four cryocoolers. The telescope mirrors and the mirror and instrument support structure are made of beryllium for excellent mass to stiffness and thermal conductance ratios. While this adds some cost to the fabrication, overall it decreases complexity because it creates an isothermal $4.5 \mathrm{~K}$ environment, which is beneficial to the telescope background stability and stability of telescope figure.

\subsection{Baffle (4.5 K)}

The baffle (Fig. 5) serves to block incoming light, shielding the mirrors. For stiffness at launch, the baffle is a 38-mm-thick aluminum honeycomb core panel. It is coated on the mirror-side with Ball Infrared Black (BIRB) or similar non-reflective coating, and the exterior is shiny aluminum. The baffle is mounted to the primary mirror support structure; however, due to material thermal contraction mismatch between the Baffle and the support structure, radially mounted titanium flexures are used at the interface to allow for deflections during cool down. Thermal straps in parallel with the flexures provide thermal contact through which the baffle is cooled.

\subsection{Thermal Shield (20 K)}

Mounted to the inside of the barrel is a light-weight $20 \mathrm{~K}$ thermal shield. The shield is composed of two double aluminized kapton (DAK) layers sandwiching a thin-aluminum foil with high 

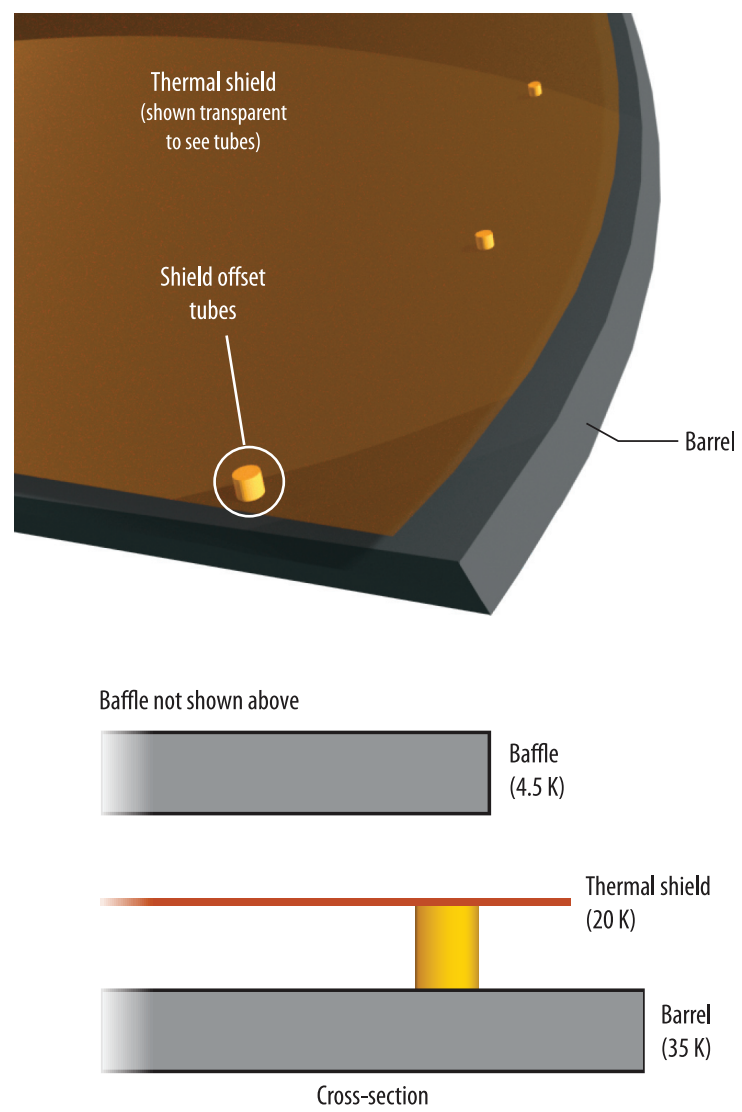

Fig. 6 Schematic of mounts of $20 \mathrm{~K}$ shield to the inside of the $35 \mathrm{~K}$ barrel.

conductance. This configuration is robust against launch loads and has very small $(<0.5 \mathrm{~K})$ temperature gradients. A similar shield is used for the MIRI instrument on JWST and has been proven thermally ${ }^{5}$ and structurally. The shield is offset from the surface of the barrel by $63 \mathrm{~mm}$ using thin-wall Vespel SP-1 tubes spaced $\sim 1 \mathrm{~m}$ apart (see Fig. 6). The shield limits the radiation from $35 \mathrm{~K}$ impinging on the $4.5 \mathrm{~K}$ surfaces. This shield is cooled by the second stage of the cryocoolers with heat exchangers positioned on the hexapod legs.

\subsection{Barrel (35 K)}

The barrel is another thermal shield comprised of cylindrical and conic sections. It is a $38-\mathrm{mm}-$ thick aluminum honeycomb core panel with high-purity aluminum face sheets on top of ordinary 6061 aluminum to provide rigidity and minimize thermal gradients. The bottom of the barrel tapers to the $35 \mathrm{~K}$ Deck. Spacecraft bipods support the $35 \mathrm{~K}$ deck from the cryogenic payload module (CPM) base as shown in Fig. 5. The CPM base provides the CPM with structural integrity and mounts to the top of the spacecraft. Access doors are located around the barrel for instrument access and integration.

The cone helps provide stiffness to the support of the cylindrical section. Bonded in the cone at the $35 \mathrm{~K}$ deck interface is a robust ring to mount to the $35 \mathrm{~K}$ deck. To further stiffen this structure, titanium struts support the barrel at the cone-cylinder interface from the base. These struts are 180-mm diameter tubes with $2.3-\mathrm{mm}$ wall thickness. The strut end fittings are also titanium and hinged at each end to allow for thermal deflection of the barrel.

Referring to Fig. 5, Fig. 5(a) shows the barrel. The left side of the barrel faces deep space, is painted black and serves as the $35 \mathrm{~K}$ radiator. The inside of the barrel faces the outside of the $20 \mathrm{~K}$ thermal shield. The inside of the barrel is actually shiny aluminum (although shown as black) for low emissivity. The right side faces the relatively hot inner sunshield and is also shiny aluminum. The low emissivity (shiny) surface is realized through either polishing the aluminum 
surface or applying a vapor-deposited aluminum-coated mylar membrane directly on the rough aluminum surface.

\subsection{Bipod Support Structure}

The barrel is supported off the warm spacecraft by Ti6Al4V struts. The supports from the barrel to the telescope and baffle are carbon composite, which has the best stiffness to thermal conductivity ratio in this temperature range $(35 \mathrm{~K}$ to $4.5 \mathrm{~K})$. The two assemblies are joined together at a $35 \mathrm{~K}$ deck at the base of the barrel. This design provides excellent stiffness against the launch environment. Thin disks are inserted into the tubes to prevent direct coupling of radiation within the tube to the cold $35 \mathrm{~K}$ barrel. Radiation-intercepting shields, forming the base of the two sunshields, are strategically located along the titanium hexapods. A $70 \mathrm{~K}$ heat intercept (not shown) is also located along each titanium tube to limit the heat load to the $35 \mathrm{~K}$ barrel. The supports from the $35 \mathrm{~K}$ deck to the telescope are carbon composite (M55J) bipods. These bipods have a $20 \mathrm{~K}$ heat intercept located strategically along the length of each tube.

\subsection{Sunshields}

Two sunshields (inner sunshield, outer sunshield) are deployed from the base. (Figs. 4 and 7). The two sunshields have similar designs. The shielding material is DAK, with the Sun-facing side of the outer layer protected by a composite silver coating [Goddard composite coating (GCC)], as described in the thermal analysis section. Each sunshield is mounted to the top-side of the CPM base, with the inner sunshield mounted higher than the outer sunshield. Each shield has a horizontal telescoping arm fixed to the base to move the shields radially outward from the barrel after launch. The arms are retracted and locked before launch. In the stowed configuration, each shield is retracted inward, allowing the shield material to wrap around the barrel. Wrapping the barrel protects the shiny aluminum surfaces from direct sunlight on-orbit, which could otherwise overheat the coatings on the aluminum structure. A vertical post is located at the end of horizontal arm of the inner and outer sunshields. These vertical posts are lightweight aluminum tubes extending from the arms vertically to the end of each shield. The outer sunshield has an additional, closely positioned post that can straddle the inner sunshield post so the outer sunshield can be fully retracted to the barrel in the stowed condition. Extending laterally on each side from the posts are flexible, small-diameter, hollow, carbon fiber composite rods. The design uses a pair of rods at the bottom, middle, and top of the shields. The rods are curved to maintain the curved shield shape upon deployment. For stowage, these rods are bent under preload to conform to the shape of the barrel.
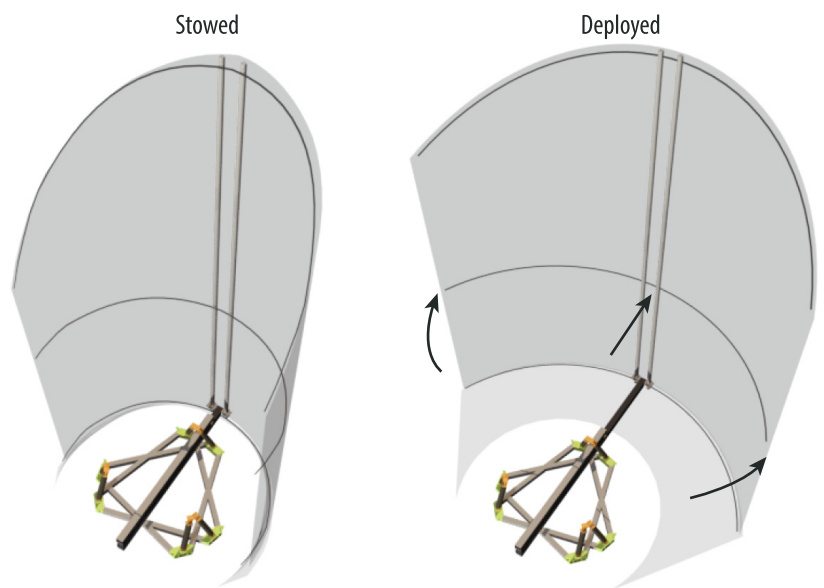

Fig. 7 The two-layer sunshield is deployed by releasing the layers from the barrel and pushing them outward from the base. Only the outer layer is shown for clarity. 
The inner and outer sunshield DAK is fixed along the masts and flexible rods. The inner and outer sunshield extends radially inward at the bottom of each shield to protect from solar and spacecraft thermal inputs. Each vertical, curved shield is attached to its respective flat, fixed sunshield fixed along the bus bipods at thermally strategic locations. The outer sunshield has a separate section that closes out to the top edge of the spacecraft bus preventing solar hot-spots between the spacecraft and shields.

The deployment scheme is simple with very few actuators and moving parts. Due to its simplicity and light weight, the deployment can be demonstrated on the ground without the need for off-loading. The sunshield deployment system uses springs to force out the telescoping arms of each shield assembly and uses the stored energy in the flexible rods to pull the shield material into its final shape. Two restraint systems are used to hold the deployment system in the stowed launch configuration. A launch lock device secures the retracted telescoping arm inside the fixed portion of each main horizontal deployment arm. Kevlar strings tensioned through the hollow flexible rods are used to hold the rods to the shape of the barrel. To release the sunshields, the strings are released and then retracted with a spring-loaded reel. The arm launch lock releases the telescoping arm section that places each shield at the intended radius from the barrel. A short video of the Origins deployments can be seen along with other Origins materials. ${ }^{1}$

The flat inner fixed sunshield and outer fixed sunshield close out the inner and outer sunshields, respectively, at the bottom, protecting the cold payload from the warm spacecraft. They are mounted to the bus bipods at the appropriate height to achieve proper thermal effects. The flat sunshields' structure comprises aluminum tube sections that hoop around the bipods and at the major shield diameter with spokes attaching the two hoops. The support structure is covered in DAK. The fixed shields do not change position and are attached to the deployable shields making a continuous thermal shield.

The telescoping structure is notional and has not yet been analyzed for deployed frequency and any interaction with the attitude control system. Its deployed mass is about $20 \mathrm{~kg}$ compared to an observatory mass of about 14 metric tons, but a low torsional mode about the support may still adversely affect the control system. In this case, a stiffer support utilizing two telescoping beams for each shield may be employed.

\section{Thermal Design and Analysis}

To keep telescope emission lower than or comparable to the sky background, the telescope is cooled to $\sim 4.5 \mathrm{~K}$. The cooling system is a combination of passive cooling provided by a twolayer deployed sunshield, a single stage radiator at $35 \mathrm{~K}$, and four high-TRL mechanical cryocoolers ${ }^{4}$ in parallel. The coolers are sized to be relatively easy extensions of current technology and provide $>100 \%$ margin on the estimated cooling load (see Table 1). The cryocooler candidates are currently TRL 4 or 5 and have technology development plans to reach TRL 6 by PDR. The lightweight two-layer sunshield is positioned between the telescope and Sun/Earth/ Moon. The layers are reflective in the Sun-ward direction, but effectively black in the perpendicular direction, cooling to deep space. The sunshield layers are designed so the outer sunshield only sees the inner sunshield, and the inner sunshield only sees the outer sunshield and the $35 \mathrm{~K}$ barrel. The Sun-facing side of the outer sunshield is coated with $\mathrm{GCC}^{6}$ tuned for high reflection of sunlight while still having a high emittance in the mid infrared wavelengths. This material has flown as the outer layer on a multi-layer insulation blanket on many previous spaceflight missions, including the Hubble Space Telescope New Outer Blanket Layer, the Geostationary Operational Environmental Satellite, and the Solar Dynamics Observatory.

Inside the $35 \mathrm{~K}$ barrel, the cryocoolers provide a $20 \mathrm{~K}$ stage for intermediate cooling of the structure and wires. Together, the cryocoolers span the $300 \mathrm{~K}$ to $4.5 \mathrm{~K}$ temperatures of the spacecraft to telescope/instruments with two intermediate cooling intercepts. Notionally, these cooling intercepts are at $\sim 70 \mathrm{~K}, \sim 20 \mathrm{~K}$, and $4.5 \mathrm{~K}$. Each of the four cryocoolers can provide $50 \mathrm{~mW}$ of cooling at $4.5 \mathrm{~K}, 100 \mathrm{~mW}$ at $20 \mathrm{~K}$, and $5 \mathrm{~W}$ at $70 \mathrm{~K}$. A heat flow map shows the thermal performance of the CPM in Fig. 8 .

The observatory thermal model (Fig. 9) was developed to simulate Origins end-to-end thermal performance. This figure is colored by thermal control coating. The integrated model 
Table 1 The heat flows to each of the three cryocooler stages shows a margin of at least $100 \%$ at each temperature. Due to the complex interaction between radiation, conduction and radiation to space, only the totals are given for the $70 \mathrm{~K}$ heat loads. Heat loads from harnesses are not currently intercepted at $20 \mathrm{~K}$, but they will be in a future iteration.

\begin{tabular}{|c|c|c|c|c|c|}
\hline $\mathrm{T}(\mathrm{K})$ & Item & Heat load (mW) & Total & Capability & Margin (\%) \\
\hline \multirow[t]{4}{*}{4.5} & Harnesses & 36 & $90 \mathrm{~mW}$ & $200 \mathrm{~mW}$ & 122 \\
\hline & Dissipation & 31 & & & \\
\hline & $4 \mathrm{~K}$ Bipods & 17 & & & \\
\hline & Radiation & 5 & & & \\
\hline \multirow[t]{3}{*}{20} & $\begin{array}{c}\text { Harnesses } \\
\text { Shield suspension }\end{array}$ & $\begin{array}{c}0 \\
28\end{array}$ & $193 \mathrm{~mW}$ & $400 \mathrm{~mW}$ & 107 \\
\hline & $4 \mathrm{~K}$ Bipods & 134 & & & \\
\hline & Radiation & 33 & & & \\
\hline \multirow[t]{2}{*}{70} & $\begin{array}{l}\text { Harnesses } \\
\text { Supports }\end{array}$ & & $9.38 \mathrm{~W}$ & $20 \mathrm{~W}$ & 113 \\
\hline & Radiation & & & & \\
\hline
\end{tabular}

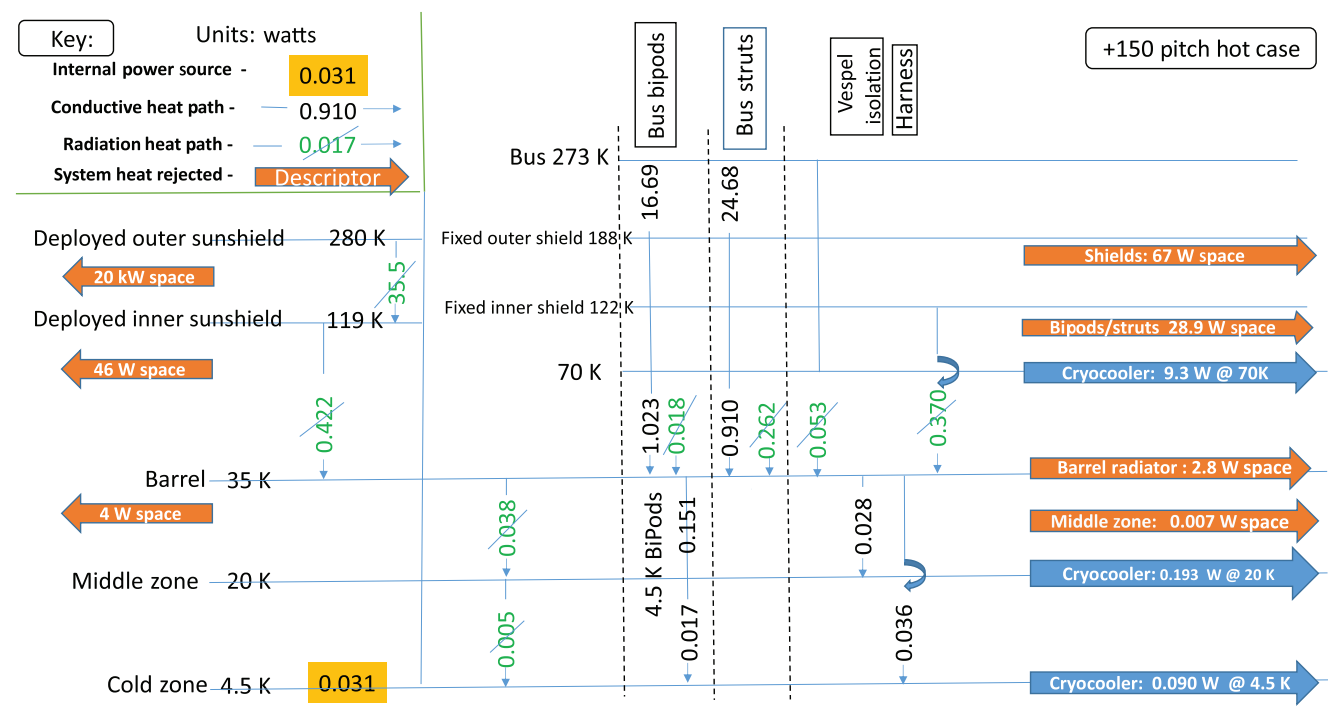

Fig. 8 Origins heat flow map shows that multiple stages of cooling results in a reasonably small heat load to the cryocooler and radiative cooler stages.

includes both bus and CPM hardware elements in sufficient detail to accurately simulate key heat loads and heat transfer paths. The thermal model employs 7170 nodes to simulate Origins thermal performance.

Multiple stages of cooling intercept heat from warmer temperatures resulting in a modest heat load $(<100 \mathrm{~mW})$ at $4.5 \mathrm{~K}$. Furthermore, staged cooling offers relative immunity to external heat load disturbances. Isothermal conditions are provided at each stage by use of thermally conductive materials (i.e., beryllium and aluminum) with a shiny vapor deposited aluminum (VDA) thermal coating. Heat is easily transported to the cryocooler heat-exchangers with minimal temperature gradient as is seen in Fig. 10.

Origins applies a $100 \%$ design margin to each of the cryocooled stages, defined as (capability - heat load)/heat load. This conservative margin is standard practice for the concept 


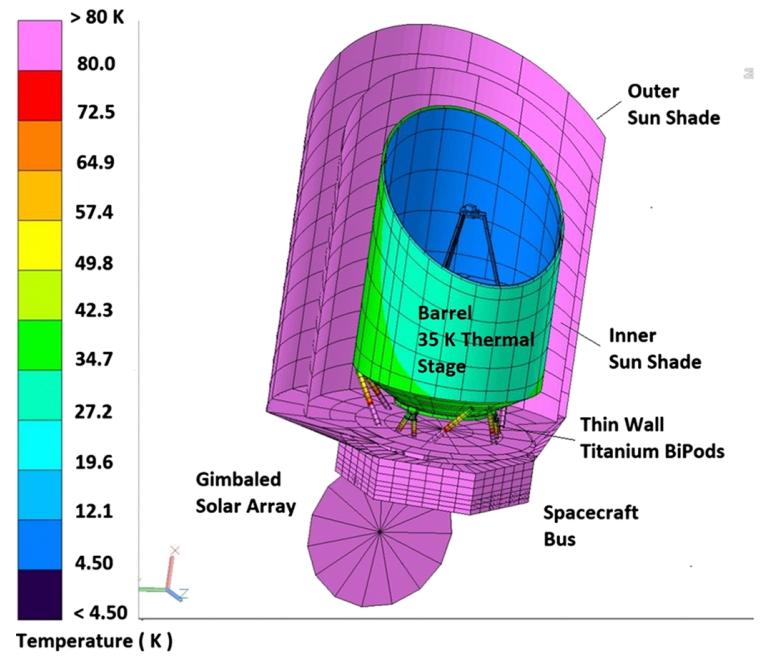

(a)

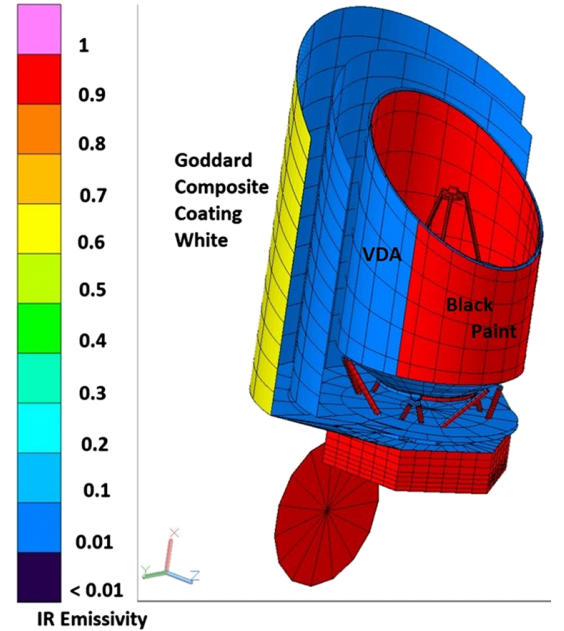

(b)

Fig. 9 (a) A Spitzer-like architecture with staged cooling, a two-layer sunshield, a deep space radiator, and cryocoolers delivers the required thermal performance with a relatively simple-tomodel design. (b) The temperature map and right shows the emissivities used to create the model.
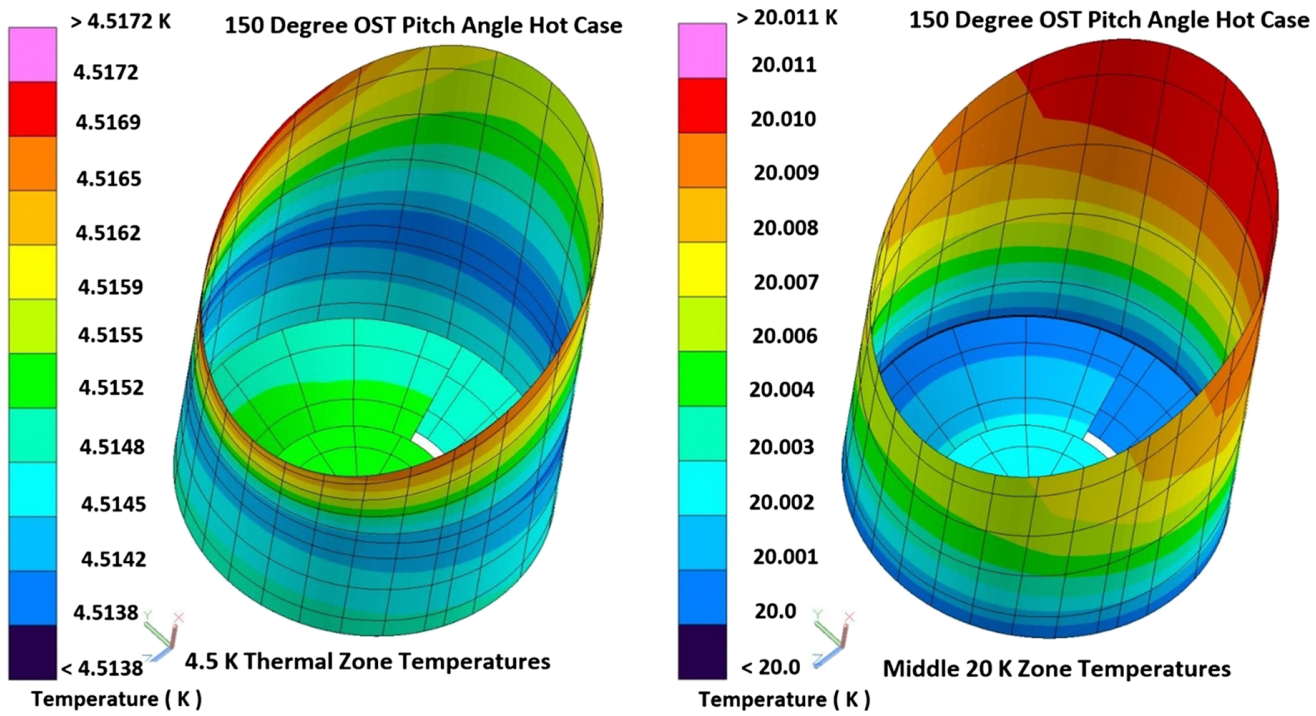

Fig. 10 The $4.5 \mathrm{~K}$ shell and $20 \mathrm{~K}$ shield temperature contours remain nearly isothermal even for the worst case, 150-degree pitch attitude.

design stage. ${ }^{7}$ Combining this margin with the cooling capability at each stage establishes $100 \mathrm{~mW}$ at $4.5 \mathrm{~K}, 200 \mathrm{~mW}$ at $20 \mathrm{~K}$, and $10 \mathrm{~W}$ at $70 \mathrm{~K}$ as required heat load maxima for the baseline design. Tables 2-5 summarize thermal model hot and cold design refrigeration performance results for the full Observatory pitch angle range of $85^{\circ}$ to $150^{\circ}$ with respect to the Sun direction. The model shows that the $100 \%$ margin requirements for refrigeration are satisfied for all cases. Somewhat counterintuitive is that the hot case occurs when the observatory points away from the Sun. This is due to the closer spacing of the shields below the bottom of the barrel. Also, it is worth noting that because the cryocoolers provide the critical temperatures of the telescope and baffle, no temperature change or temperature gradient change is observed in these critical elements. It is worth noting the very small gradients in the $4.5 \mathrm{~K}$ and $20 \mathrm{~K}$ zones in Fig. 10 .

Figure 11 shows that even the barrel, with a large heat input and radiative cooling is relatively isothermal. 
Table 2. Origins uses well-documented thermal optical properties for hot and cold design cases.

\begin{tabular}{|c|c|c|c|c|}
\hline \multicolumn{5}{|c|}{ Design hot and cold thermal optical properties } \\
\hline & \multicolumn{2}{|c|}{ Cold cases } & \multicolumn{2}{|c|}{ Hot cases } \\
\hline & \multicolumn{2}{|c|}{$\mathrm{BOL}$} & \multicolumn{2}{|c|}{ EOL } \\
\hline & Alpha & Emittance & Alpha & Emittance \\
\hline \multicolumn{5}{|l|}{ Material } \\
\hline Kapton 3mil & 0.45 & 0.80 & 0.57 & 0.93 \\
\hline Solar array cell & 0.60 & 0.76 & 0.82 & 0.76 \\
\hline GCC white on kapton & 0.07 & 0.70 & 0.17 & 0.64 \\
\hline Z93C55 white paint & 0.21 & 0.91 & 0.37 & 0.88 \\
\hline BIRB black paint & 0.99 & 0.88 & 0.99 & 0.88 \\
\hline \multicolumn{5}{|c|}{ Cold zone VDA assumptions } \\
\hline VDA warm & 0.12 & 0.0250 & 0.12 & 0.0250 \\
\hline VDA_150 K & 0.12 & 0.0150 & 0.12 & 0.0150 \\
\hline VDA_100 K & 0.12 & 0.0125 & 0.12 & 0.0125 \\
\hline VDA_30 K & 0.12 & 0.0100 & 0.12 & 0.0100 \\
\hline
\end{tabular}

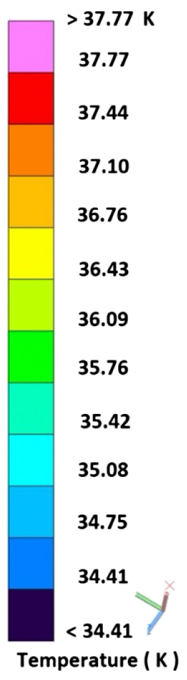

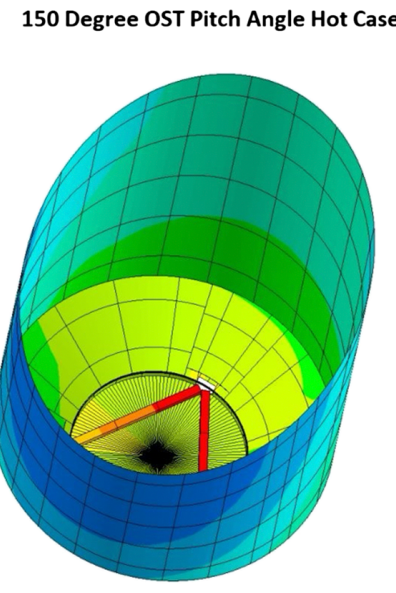

Barrel Temperature Contours

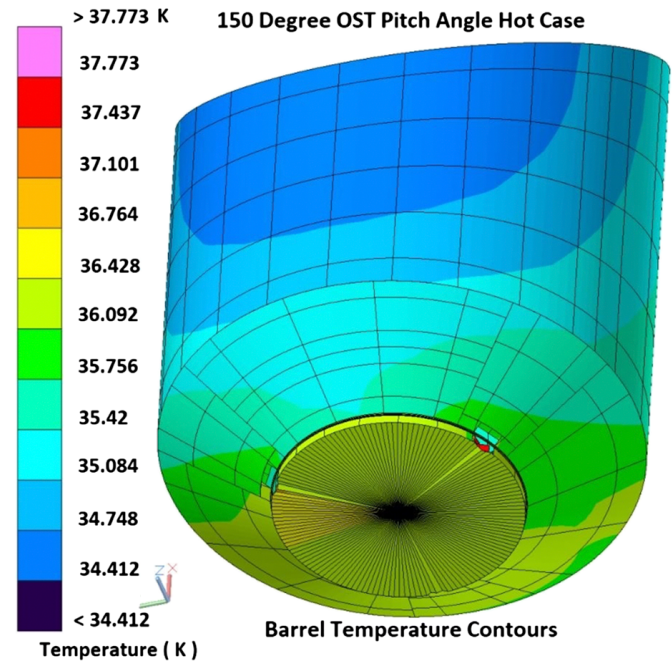

Fig. 11 The barrel temperature contours show that the barrel temperature remains below $36 \mathrm{~K}$ and nearly isothermal in the worst-case attitude $\left(150^{\circ}\right.$ pitch) over nearly all of its surface.

Hot and cold thermal simulations were performed to bound thermal performance for the full Observatory attitude/pitch range. Bounding cold/hot parameters include the solar irradiance at SEL2 (1291 to $1421 \mathrm{~W} / \mathrm{m}^{2}$ ), beginning of life (BOL)/end of life (EOL) thermal optical properties, are used to calculate heat loads and temperatures. In the case of BIRB, recent precision emissivity measurements ${ }^{8}$ at $35 \mathrm{~K}$ exceed those in the Table 2 , so the predicted barrel radiator performance is conservative. The worst case heat loads are used in Table 1. The worst-case results for the sunshield temperatures are shown in Fig. 12. 


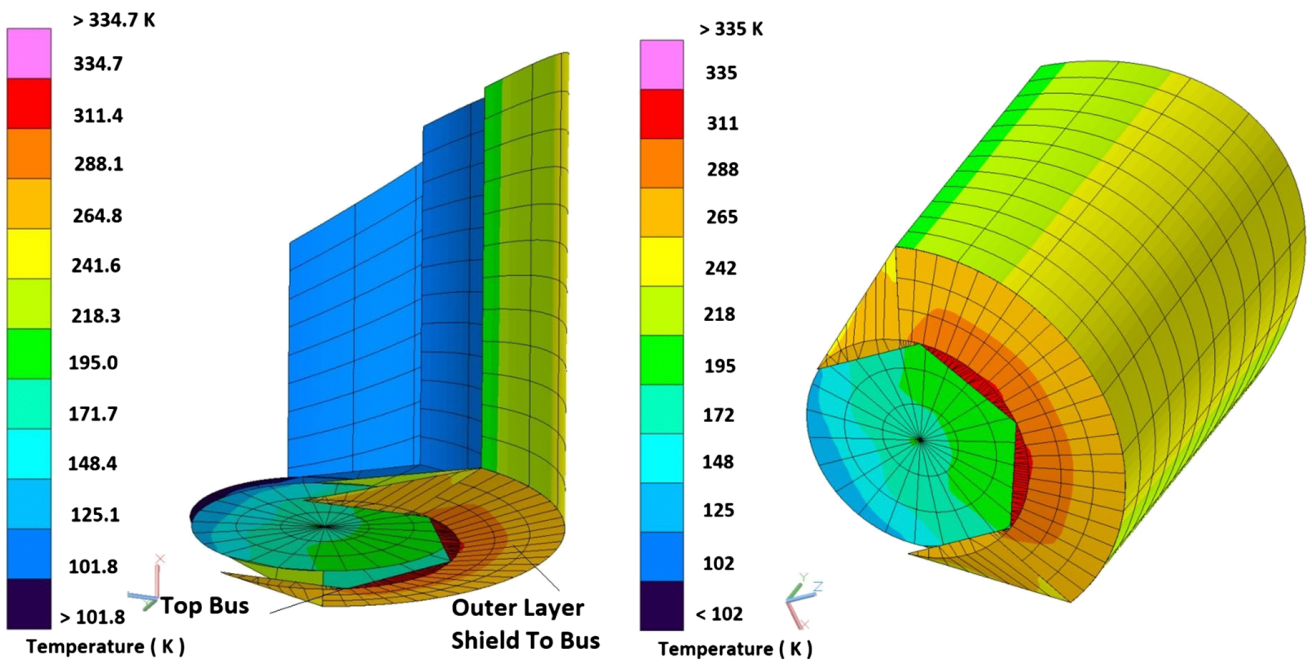

Fig. 12 The outer sunshield with GCC maintains room temperature or lower for all FOR over nearly all of its surface.

Table 1 shows the calculated heat flows to each of the cryocooler stages. At first glance it looks coincidental that each of the stages has close to the same heat load margin. What is actually taking place is the balancing of heat loads by strategic interception of heat along the struts and harnesses, and the tradeoffs that can be made within the cryocoolers themselves between the fraction of heat lifted between the $20 \mathrm{~K}$ and $4.5 \mathrm{~K}$ stages. Note that there is a very small cooling at $20 \mathrm{~K}$ from radiation to space.

\section{Summary}

Early trades performed on the Origins configuration emphasized simplicity, minimum deployments and ease of modeling. ${ }^{9}$ The same is true of the Origins cryo-thermal system. The resulting design is relatively easy to analyze. With the simple, lightweight shields, Origins would be able to be tested in its flight configuration in an existing thermal vacuum chamber. ${ }^{10}$ Thermal analysis shows that the design meets its targeted goals for temperature and heat loads and, simultaneously provides near isothermal conditions on sensitive surfaces independent of the pointing angle.

\section{Acknowledgments}

The authors are grateful to the many institutions that sponsored and contributed to the successful Origins Space Telescope mission concept study.

\section{References}

1. “Origins Space Telescope Mission Concept Study Final Report," https://asd.gsfc.nasa.gov/ firs/docs/.

2. M. W. Werner et al., "The Spitzer Space Telescope mission," Astrophys. J. Suppl. 154, 1-9 (2004).

3. J. Arenberg et al., "Alternate architecture for the Origins Space Telescope," J. Astron. Telesc. Instrum. Syst. 7(1), 011006 (2021).

4. M. DiPirro et al., "Cryocooling technologies for the Origins Space Telescope," J. Astron. Telesc. Instrum. Syst. 7(1), 011008 (2021).

5. J. Tuttle et al., "Thermal properties of double-aluminized kapton at low temperatures," in Adv. Cryogenic Eng. Mater. Trans. ICMC, Vol. 54, p. 34 (2008).

6. M. Hasegawa, Provisional patent. GCC is composite silver, CCAg, with the appropriate thickness overcoat has an absorptance in the near UV/visible of 0.10 , and an emittance in the mid IR of 0.68 . 
7. M. Donabedian, Ed., AIAA Spacecraft Thermal Control Handbook, Vol. 2 Cryogenics, pp. 470-473, American Institute of Astronautics/Aerospace Press (2003).

8. J. Tuttle et al., "The area-density-dependence of Ball IR Black's low-temperature emissivity," Cryogenics, 64, 240 (2014).

9. D. Leisawitz et al., "The Origins Space Telescope: trades and decisions leading to the baseline mission concept, and future study topics," J. Astron. Telesc. Instrum. Syst. 7(1) (2021).

10. S. Petro, "Origins Space Telescope integration and testing," J. Astron. Telesc. Instrum. Syst. 7(4), 041502 (2021).

Michael DiPirro received his $\mathrm{PhD}$ in low-temperature physics from the State University of New York at Buffalo, and a one-year National Research Council postdoctoral fellowship at the National Bureau of Standards, and then he joined NASA Goddard in 1980. He has worked on a number of astrophysics missions over the last 40 years, including Cosmic Background Explorer (COBE), ASTRO-E, -E2, and -H, Spitzer, Wide Field Infrared Explorer (WIRE), Wide Field Infrared Survey Explorer (WISE), and JWST. Between COBE and ASTRO-E, he was the principal investigator on the Superfluid Helium On-Orbit Transfer Flight Demonstration and Co-I on a Cross Enterprise Technology Development Program to develop a new type of adiabatic demagnetization refrigerator. He is the lead technical engineer on the Origins Space Telescope Study and is also currently the subject matter expert for cryogenics on the X-Ray Imaging and Spectroscopy Mission/resolve X-ray microcalorimeter instrument.

Biographies of the other authors are not available. 